\title{
Costos y rentabilidad del proceso de producción apícola en México ${ }^{1}$
}

Miguel A. Magaña
Magaña
Instituto Tecnológico de Conkal,
Yucatán.mmagana@gmail.com

Carlos E. Leyva Morales Universidad Autónoma de Yucatán.clmoral@tunku.uady.mx

${ }^{1}$ Parte del proyecto de investigación "Generación de tecnologías para la obtención de productos inocuos de la colmena y su competitividad en el mercado" financiado por el Conacyt, clave 12699.

\section{Resumen}

En este estudio se buscó determinar y analizar el nivel y la estructura del costo y la rentabilidad del proceso de producción de la miel de abeja en los siete principales estados productores de México. Se encontró que la estructura de los costos de producción de la miel se compone mayoritariamente por el costo variable $(67.1 \%)$ cuyos principales rubros son la erogación para el pago de mano de obra y compra de insumos alimenticios, mientras que en el caso de los costos fijos (32.9\%) el principal valor lo constituyó la depreciación en infraestructura $(23.3 \%)$. Esta composición del costo total tiene relevancia en la administración de la producción apícola nacional, pues las variaciones en los precios hacia la baja afectan en forma más sensible a los apicultores cuyo costo fijo medio es menor. Por su parte, la principal causa que puede provocar la variación en el nivel de la rentabilidad de las explotaciones apícolas la representa el nivel del ingreso por la venta de miel, el cual determina el precio que se le paga al productor mexicano, que depende del mercado donde realice la transacción. Finalmente, se encontró que la rentabilidad por colmena en México asciende a 158 pesos y a 305.3 pesos por cada mil pesos invertidos en activos fijos, en tanto que la relación beneficio-costo indica una rentabilidad de 38 centavos por cada peso invertido; esta actividad es rentable porque lo invertido supera la recuperación que se obtendría en pagarés de renta fija.

Palabras clave: apicultura, proceso de producción, costos y rentabilidad. 


\title{
Costs and profitability of beekeeping production process in Mexico
}

\begin{abstract}
This study aims to determine and analyze the level, cost structure and profitability of the honey bee production process in the seven main producing states in Mexico. It was found that the structure of honey production costs is mostly composed of the variable cost $(67.1 \%)$, its main values being labor payment and purchase of food inputs, while in the case of fixed costs $(32.9 \%)$, the main value was infrastructure depreciation $(23.3 \%)$. This cost composition of the total cost has relevance in the administration of national beekeeping production since downtrend variations in prices mainly affect beekeepers whose average fixed cost is lower. On its part, the main cause that can allow the variation in the level of profitability of the apiculture holdings in Mexico is represented by the level of income from the sale of honey, which is determined by the price paid to the Mexican producer depending on the market where the transaction is carried out. Finally, it was found that profitability per hive in Mexico is 158 pesos and 305.3 pesos for every thousand pesos invested in fixed assets, while the cost-benefit ratio indicates a profitability rate of 38 cents for each peso invested; this activity is profitable since the return on investment goes well beyond that obtained from debt notes.
\end{abstract}

Keywords: beekeeping, production process, cost and profitability.

\section{Introducción}

La apicultura en México es una actividad milenaria de gran relevancia social y económica en el sector primario debido a que ha representado una fuente de empleos, ingresos y de divisas (Güemes et al., 2003; Tiatrini, 2002). Por ejemplo, en materia de empleo, si se considera el número de unidades de producción apícola que se reporta en el VII Censo Nacional Agropecuario y Forestal $(33,981)$ y la cantidad estimada de mano de obra que se requiere en promedio por apiario, se puede inferir que la apicultura demanda aproximadamente 2.2 millones de jornadas laborales al año en su fase de producción primaria, lo cual representa una importante fuente de empleos y de ingresos por pago de salarios en el medio rural, pero por la naturaleza del proceso de producción el trabajo en los apiarios es en su mayor parte de carácter estacional.

La importancia de la actividad apícola se constata también por su participación relativa en el valor tanto del ingreso pecuario, del sector agropecuario y del PIB 
nacional, como por su papel de generador de divisas. En cuanto a la primera participación, ésta varió de 1.47 a $0.72 \%$ en los años 1990 y 2007, cuya disminución fue de 50.7\% (SIACON, 1980-2008). En cuanto al segundo apartado, la máxima contribución de la apicultura al ingreso del sector agropecuario (0.48\%) ocurrió en el año 2000 y en 2007 ésta fue de $0.3 \%$, que denota una disminución como la anterior. Con respecto al PIB nacional, el valor de la producción apícola representó el $0.023 \%$ en el año de 1990, disminuyó en 2007 (0.013\%), en este apartado la participación se redujo $42.4 \%$ y se debió principalmente a la disminución en el precio real de la miel de 1997 a 2007. Contrario a lo antes expuesto, la contribución de la apicultura en la captación de divisas es relevante para el subsector pecuario nacional, la cual fue en promedio de $12.4 \%$ (1995 a 2008); con respecto al sector agropecuario tal contribución fue de $0.94 \%$ (SAGARPA, 2009).

Por otra parte, y con relación al contexto mundial, las cifras de producción de miel señalan a China, Estados Unidos, Argentina y Turquía como los principales países productores, aportaron un poco más de la tercera parte de la oferta internacional en el período 1990-2007; México se ubicó en el quinto lugar como productor durante los primeros años de la década de los noventa, pero a partir de la segunda mitad de ésta pasó al sexto lugar, su participación en dicha oferta ha oscilado de 3.6 a 5.6\%. En tanto que en materia de comercio, los principales exportadores para el período 1990-2007 fueron Argentina, China, México, Alemania y Hungría, concentrando en promedio el $64 \%$ del total exportado; por otro lado, los cuatro principales países importadores fueron Estados Unidos, Alemania, Japón y Reino Unido, concentrando aproximadamente el $66 \%$ del total mundial. México canalizó el $73.3 \%$ del total de sus exportaciones a tres de ellos en el año 2005, destacando en ese año el 50.6\% que se envío a Alemania, mientras que hacia Estados Unidos solo canalizó el 7.2\% (FAOSTAT, 2009).

La evolución que ha seguido la importancia económica de la actividad apícola nacional se debe a que en ésta se ha tenido que enfrentar problemas de diversa índole que han afectado el nivel de su productividad y el de la rentabilidad, lo cual ha influido en forma determinante sobre su desarrollo. Las causas estructurales de la problemática y sus consecuencias sobre la producción apícola se pueden agrupar en tres puntos principales:

1. Africanización y varroasis. La abeja africana, que llegó en 1986 al país procedente de Sudamérica, afectó la producción del dulce; en tanto la 
segunda, ocasionada por el ácaro Varroa jacobsoni, se encuentra actualmente diseminada en todas las regiones apícolas del país y constituye su principal problema sanitario porque afecta a la colonia de abejas en todos sus estadios de desarrollo — cría sellada, abierta e individuo adulto- (www.InfoAgro. com, 2005). Este problema elevó los costos de producción y redujo el rendimiento.

2. Comercialización externa. La pérdida constante de competitividad de la miel mexicana en el mercado mundial no sólo se explica por los elevados costos de producción, el difícil acceso al crédito y el atraso tecnológico, sino también por la carencia de una práctica exportadora profesional. Ello explica la sustitución de la miel mexicana del mercado estadounidense por la proveniente de China y Argentina.

3. Descapitalización de la actividad. Esta situación se asocia a la disminución de las exportaciones de miel y a las dificultades económicas y financieras del país, lo que se refleja en la falta de recursos y encarecimiento del crédito interno. La descapitalización ha limitado la adopción de nuevas prácticas tecnológicas en el subsector, lo cual se manifiesta en el bajo rendimiento por colmena (www.agronet.com.mx).

Además de los tres puntos señalados, existen otros elementos que afectan en forma negativa a la apicultura como la falta de una legislación federal adecuada y actualizada que regule eficazmente la actividad, la desvinculación e inoperancia de las diversas organizaciones de productores, los escasos canales de comercialización directa entre el productor y el consumidor (www.agronet.com.mx), el bajo consumo per cápita de miel (300 g.) en relación con los significativos volúmenes de producción y la exigencia de los países importadores por productos inocuos y de mayor calidad.

Con el fin de conocer la magnitud de las variables relacionadas con el estado de resultados de la actividad apícola nacional, la hipótesis general del presente trabajo fue que la rentabilidad que obtienen los apicultores en México no está garantizada debido a que los costos de producción aumentan constantemente, mientras que los rendimientos por colmena se mantienen sin variación y los precios de venta están sujetos a la cadena comercial, situación que provoca que éstos no obtengan los ingresos suficientes. 


\section{Metodología y criterios en el proceso de estimación}

En esta investigación se utilizó como marco metodológico y teórico básico para la evaluación económica del sistema o proceso de producción apícola el enfoque del presupuesto tipo empresa propuesto por Kay (1990). Este procedimiento sirvió de base para el cálculo de la rentabilidad de la actividad primaria y permitió llevar a cabo una mejor comparación de las utilidades por empresas alternativas y competitivas.

Para obtener la información directa o de campo se empleó una encuesta por muestreo estadístico estratificado. El instrumento principal fue una cédula de entrevista cuya estructura comprendió las partes relativas a la infraestructura y capacidad de producción del apiario, los gastos derivados de diversas erogaciones y los ingresos por venta. Con el fin de realizar la encuesta a los productores, y que está representará las principales regiones apícolas, se identificó, como primera fase, a los siete principales estados productores de miel del país en el periodo 1995-2007, los cuales fueron Yucatán, Campeche, Quintana Roo, Chiapas, Veracruz, Guerrero y Jalisco. Como segunda fase, los productores fueron elegidos al azar en los diferentes municipios de las regiones estatales considerados de importancia apícola; la muestra preliminar inicial se asignó considerando tanto la importancia relativa de cada estado como el número de productores, el total de cédulas para tal muestra fue de 1,500 aproximadamente (más menos 50); esto se debió a que no existe en la literatura estimaciones de varianza asociadas al muestreo particular que permitiese la definición más eficiente de la muestra preliminar.

La variable fundamental asociada al muestreo fue la productividad de miel por colmena y se consideró a ésta debido a que es importante tanto en los costos de producción, ingresos y rentabilidad. La fórmula de muestreo para determinar el tamaño de la muestra final fue el correspondiente al estratificado (Scheaffer y col., 1987), en este caso se contempló como estrato o segmento a cada estado. El límite de error de estimación se consideró como el 3.5\% (1 kg) del valor medio de productividad por colmena en la muestra preliminar $(29.1 \mathrm{~kg})$. La asignación de productores por estrato se realizó considerando la importancia de cada estado como productor de miel (1995-2007). La muestra final de productores se presenta en el siguiente cuadro. 


\section{Cuadro 1}

Información empleada en la determinación de la muestra final

\begin{tabular}{lccccc}
\hline \multicolumn{1}{c}{ Estado } & $\begin{array}{c}\text { Unidades } \\
\text { de Prod. }\end{array}$ & $\begin{array}{c}\text { Varianza } \\
\text { Product }\end{array}$ & $\begin{array}{c}\text { Ponderador } \\
\text { por V. Prod. }\end{array}$ & $\begin{array}{c}\text { Muestra } \\
\text { Preliminar }\end{array}$ & $\begin{array}{c}\text { Muestra } \\
\text { Final }\end{array}$ \\
\hline CAMPECHE & 5,067 & 86,0450 & 0.162 & 375 & 100 \\
CHIAPAS & 2,324 & 90,1896 & 0.088 & 117 & 55 \\
GUERRERO & 961 & 95,5761 & 0.112 & 80 & 69 \\
JALISCO & 727 & 117,5915 & 0.157 & 182 & 97 \\
QUINTANA & 3,117 & 68,9348 & 0.077 & 163 & 48 \\
ROO & 1,569 & 184,8010 & 0.146 & 212 & 90 \\
VERACRUZ & 11,019 & 137,5735 & 0.257 & 410 & 159 \\
YUCATÁN & & & 1,000 & 1,539 & 618 \\
TOTAL & 24,784 & & & & \\
\hline
\end{tabular}

La encuesta a los apicultores se realizó durante las temporadas de cosecha 20072008 y la información obtenida fue registrada y procesada en una hoja de cálculo del programa excel. La estimación de los parámetros de la estadística descriptiva o, en su caso, la correlacional entre variables, se realizó con apego a lo propuesto en la literatura especializada (Stevenson, 1981).

Para determinar la relación que existe entre la rentabilidad y los costos e ingresos, se empleó un modelo de regresión lineal múltiple en el cual se consideraron como variables explicativas los principales elementos que constituyen cada uno de los dos componentes de la rentabilidad. Los parámetros estimados de la regresión $\left(\mathrm{R}^{\wedge} 2=0.821564486\right)$ se presentan en la siguiente ecuación:

$$
\begin{aligned}
& \Pi=-264.5101018+22.49694552 \mathrm{PV}+14.99436203 \mathrm{PRO}-12.80438323 \mathrm{PA} \\
& \begin{array}{lll}
(42.63127841) & (0.966514513) \quad(0.537246697)
\end{array} \\
& -0.154370055 \mathrm{SA}-0.142552421 \mathrm{TR}-1.488747649 \mathrm{DI} \\
& \begin{array}{lll}
(0.105130346) & (0.008052289) & (0.300099652)
\end{array}
\end{aligned}
$$

Donde: $\Pi=$ Rentabilidad por colmena

$\mathrm{PV}=$ Precio que recibe el apicultor por kilogramo de miel

$\mathrm{PRO}=$ Rendimiento de miel por colmena

PA $=$ Precio del azúcar por kilogramo

$\mathrm{SA}=$ Salario promedio por jornada

$\mathrm{TR}=$ Transporte por contenedor de 300 kilogramos de miel .

DI = Valor promedio por colmena de la depreciación de infraestructura 
Entre los estudios más recientes sobre la temática de los costos y rentabilidad de empresas apícolas se encuentra el trabajo realizado por Feitosa et al. (2004) donde la evaluación de la rentabilidad de los productores apícolas de las ciudades de Mombaça, Pacajús y Chorozinho fue realizada utilizando la metodología del sistema integrado de costos agropecuarios (CUSTAGRI, 1998), en el que se encontró que la producción de miel es muy rentable, que ésta registra bajos costos y que puede alcanzar altos índices de beneficios en el caso de las ciudades de Mombaça y Pacajús, como consecuencia de los mejores precios recibidos, debido a la existencia de un canal de comercialización directo y por su cercanía a la capital del estado. Por su parte, los resultados del estudio del CREEBBA (2005), en el cual se buscó presentar evidencia de la crisis de rentabilidad de los pequeños y medianos productores de Argentina y actualizar los costos para calcular el punto de equilibrio con base en una metodología generado por ellos, indicaron que el costo de producción por kilo de miel en el año 2003 era de $\$ 2.49$, mientras que en el 2005 este valor ascendió a $\$ 2.58$ (equivalentes a US \$ 0.76 y US\$ 0.86, respectivamente), en tanto que el costo variable de una colmena se incrementó en un $4 \%$ (de $\$ 93.29$ a \$96.93), dando lugar a una pérdida de rentabilidad, aunque argumentan como la causa principal de esta situación a la caída del precio al productor. El tercer estudio de referencia fue realizado por la Red Nacional de Centros de Gestión y el Instituto de Desarrollo Agropecuario de Chile (2008), que buscó identificar los indicadores productivos y económicos, así como elaborar un plan de cuentas y definir criterios comunes de registro para el rubro apícola con base en modelos propios, arrojando la existencia de una gran diferencia entre los costos de producción de un kilo de miel (US \$ 1.1) y los costos variables de una colmena en este país. Se observó un margen de utilidad mínimo, ya que el precio pagado al productor fue de US \$1.4, situación que redundó en una baja rentabilidad de los sistemas tradicionales de producción, aunque estas diferencias se debieron más a la utilización de criterios dispares de clasificación, de valoración y de reconocimiento de costos directos más que a grandes diferencias en los sistemas productivos.

\section{Costos de producción y rentabilidad de la miel en México}

Antes de iniciar este apartado es importante mencionar que la competitividad de todo sistema o proceso de producción en el mercado interno lo confirma el nivel de su rentabilidad; éste se logra al descontar al valor de la venta de cierta cantidad de producto los costos en los que se incurrió para obtenerlo. Por lo tanto, del lado del ingreso figura la productividad del proceso (producto) y la capacidad negociadora que tiene el productor o empresario para la venta (precio); del lado del costo total 
están la cantidad de insumos y servicios empleados en el proceso de producción, sus precios de compra y los valores de la depreciación de los equipos e infraestructura, esto último acorde con el nivel tecnológico del proceso de producción.

Por su parte, el nivel unitario o promedio de dicho costo en la apicultura depende de dos aspectos básicos: primero, del desempeño o eficiencia técnica del proceso productivo, que es determinado por la tecnología, la capacidad administrativa en la toma de decisiones, la disponibilidad y calidad de los recursos naturales e influencia de los factores ambientales; segundo, de la estructura del mercado de los insumos, que determina los respectivos niveles de precios. Estos aspectos adquieren una gran relevancia en la comparación de los ingresos y costos de producción al analizar la rentabilidad por regiones.

\section{Inversiones en colmenas y equipo}

Las unidades productivas que conforman la apicultura en México están integradas por las clásicas colmenas estándares, con una base o soporte que sostiene dos cuerpos; piso y piquera de madera; cámara de cría con diez cuadros en su interior; rejilla excluidora, entre tapa; techo y depósito o malario. La evaluación se realizó con dos medias alzas por colmena, con sus respectivos cuadros.

El capital que se evaluó está formado por colmenas que componen el módulo productivo en promedio, a las que se sumó el conjunto de las herramientas, equipos e instalaciones necesarios para llevar adelante esa actividad (cuadro 2).

\section{Cuadro 2}

Inversión realizada por apiario según rubro de interés

\begin{tabular}{lccc} 
Referencia & $\begin{array}{c}\text { Equipo de protección } \\
\text { y trab. } \\
(\$)\end{array}$ & $\begin{array}{c}\text { Infraestructura } \\
\text { en campo } \\
(\$)\end{array}$ & $\begin{array}{c}\text { Total de } \\
\text { Inversión } \\
(\$)\end{array}$ \\
\hline PROM EST & $1,340.5$ & $11,960.8$ & $13,301.4$ \\
CAMPECHE & $1,542.5$ & $7,914.1$ & $9,456.6$ \\
CHIAPAS & $2,838.3$ & $5,904.6$ & $8,742.9$ \\
GUERRERO & $1,782.3$ & $10,078.8$ & $11,861.1$ \\
JALISCO & $1,318.0$ & $18,216.8$ & $19,534.9$ \\
QUINTANA & $3,223.1$ & $8,342.6$ & $11,565.7$ \\
ROO & $1,002.4$ & $8,264.0$ & $9,266.4$ \\
VERACRUZ & 730.4 & $7,491.8$ & $8,222.2$ \\
YUCATÁN & & & \\
\hline
\end{tabular}

Fuente: Encuesta a productores 
En el cuadro de inversiones también se incluyen las erogaciones por la adquisición de los núcleos de abejas para instalar el apiario, generalmente compuestos por tres o cuatro cuadros que contienen miel, polen, abejas y una reina joven. El valor del terreno destinado al apiario no se incluyó debido a la baja superficie del mismo, pudiéndose obtener (aparte del utilizado en el propio campo) en calidad de préstamo o también a cambio de los servicios de polinización que realizan las abejas en los cultivos de la región.

La diferencia en el valor promedio de inversión observado por estado depende del número de apiarios en posesión del apicultor y del número de colmenas que integran cada uno de ellos (cuadro 3). Por ejemplo, cuando se tiene un solo apiario se dispone del mismo equipo de trabajo y de protección que si se tuviesen tres, por lo que la inversión total es la misma en este rubro, lo cual diferencia el valor promedio. Otro aspecto de importancia que explica la diferencia en inversión es la calidad del equipo: un extractor de acero inoxidable para cuatro bastidores tiene un valor casi cinco veces superior al de acero galvanizado.

\section{Estructura del costo de producción}

En todo sistema o proceso de producción orientado al mercado es importante la estimación y definición de la estructura de los costos de producción, pues éstos determinarán, juntamente con los ingresos, el nivel de ganancias o rentabilidad del sistema o proceso de producción.

En la figura 1 se observa que la estructura porcentual de los costos de producción de la miel en México se compone mayoritariamente por el costo variable, cuya participación relativa representa desde el $69.7 \%$ en el estado de Jalisco hasta el $49.9 \%$ en el estado de Quintana Roo. En este último estado, el costo variable y el fijo son casi de la misma magnitud y este hecho obedece principalmente a que la contratación y pago de mano de obra es bajo, al igual que la erogación en combustible; estos dos rubros disminuyen apreciablemente el costo variable. 
Figura 1

Estructura porcentual del costo de producción de miel

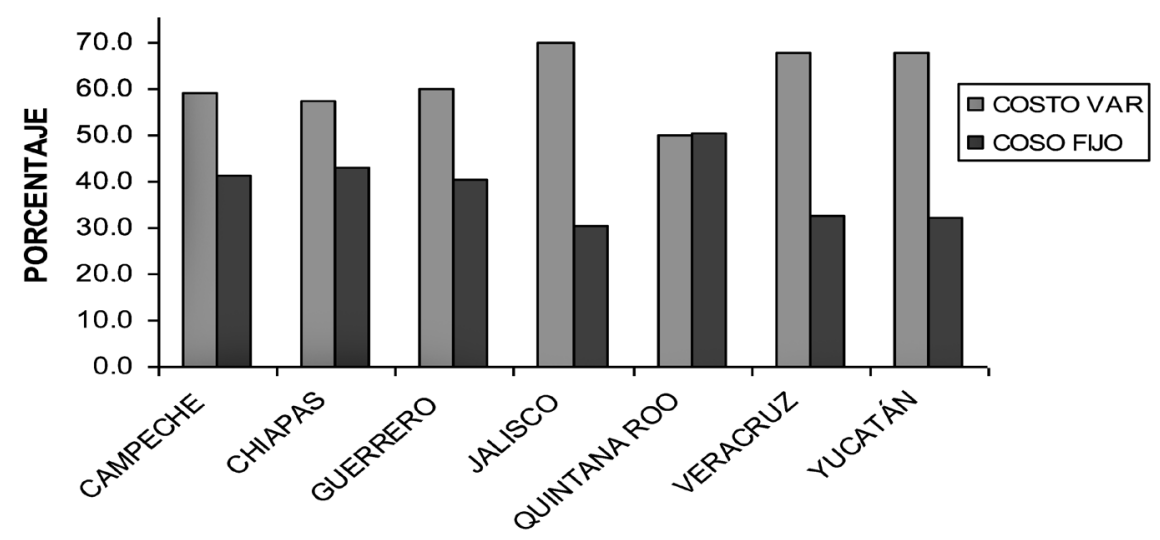

De la estructura del costo de producción total de la miel en el país, la participación relativa de las erogaciones en la compra de azúcar representó el $11.2 \%$ y el pago a la mano de obra representó el 31.2\% (cuadro 3); esta última erogación fue también la principal en el rubro de los costos variables (46.5\%) y se debe a que los apicultores nacionales tienen la necesidad de contratar mano de obra para la realización de las diversas actividades en el apiario; de ellas la principal es la cosecha.

Por otra parte, el rubro de medicamentos, como se observa del cuadro ya mencionado, representó aproximadamente el $2 \%$ en el total del costo de producción. Por estados esta erogación representó el 6.2 y 5.7\% del costo en Campeche y Yucatán, las cuales fueron las más elevadas y quizás se deba a que la varroasis aún representa un serio problema en los apiarios de dichas entidades, no así en estados como Veracruz y Guerrero, donde la erogación por este concepto no superó el $0.9 \%$.

Con respecto al costo relativo de los medicamentos, es importante mencionar que en varios estados de la República la incidencia de la varroasis ya ha sido abatida a niveles bajos. En esta reducción ha jugado un papel muy importante tanto la capacitación otorgada a los apicultores en el manejo de dicho parásito (para el control eficiente y evitar la resistencia), como los apoyos que otorgan los gobiernos estatales y federal, entre ellos la venta de medicamentos a mitad de precio, o el uso de productos alternos e inocuos para tal fin como el timol. 


\section{Cuadro 3}

\section{Costos de producción y rentabilidad de la actividad apícola por apiario}

\begin{tabular}{|c|c|c|c|c|c|c|c|c|}
\hline CONCEPTO & 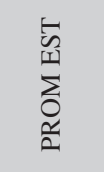 & 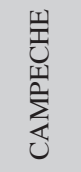 & 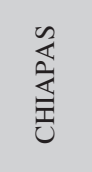 & $\begin{array}{l}0 \\
\frac{\alpha}{w} \\
\frac{1}{0} \\
\frac{1}{4} \\
0 \\
0\end{array}$ & 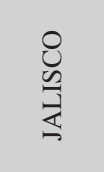 & 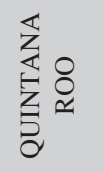 & 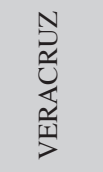 & 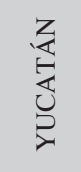 \\
\hline PROD./COLMENA $(\mathrm{kg})$ & 29.1 & 30.4 & 25.4 & 24.8 & 25.3 & 30.6 & 36.6 & 35.6 \\
\hline COLMENAS/APIARIO & 25.7 & 21.2 & 17.2 & 25.1 & 34.1 & 20.6 & 21.5 & 17.9 \\
\hline \multicolumn{9}{|l|}{ COSTO VARIABLE (\$) } \\
\hline Azúcar & 1,217 & 692 & 748 & 960 & 1,794 & 1,076 & 948 & 777 \\
\hline Otros insumos alim. & 100 & 32 & 1 & 324 & 182 & 72 & 39 & 8 \\
\hline Medicamentos & 219 & 352 & 83 & 81 & 211 & 60 & 65 & 406 \\
\hline Cera & 335 & 453 & 511 & 525 & 279 & 501 & 330 & 270 \\
\hline Mano de obra & 3,373 & 932 & 1,789 & 2,501 & 4,830 & 1,166 & 3,812 & 2,447 \\
\hline Flete & 134 & 300 & 92 & 148 & 18 & 293 & 100 & 271 \\
\hline Combustible & 1,253 & 428 & 658 & 726 & 1,958 & 341 & 1,545 & 453 \\
\hline Refacciones y mant. & 571 & 125 & 374 & 401 & 847 & 175 & 835 & 179 \\
\hline Otros & 66 & 7 & 34 & 0 & 122 & 6 & 62 & 27 \\
\hline TOTAL COST. VAR. & 7,269 & 3,320 & 4,289 & 5,665 & 10,241 & 3,689 & 7,736 & 4,836 \\
\hline \multicolumn{9}{|l|}{ COSTO FIJO $(\$)$} \\
\hline Deprecia. equipo & 707 & 453 & 1,001 & 717 & 869 & 951 & 713 & 396 \\
\hline Deprecia. infraest & 2,202 & 1,729 & 1,661 & 2,475 & 2,670 & 2,117 & 2,043 & 1,725 \\
\hline Deprecia. vehículo & 215 & 85 & 172 & 260 & 282 & 377 & 226 & 95 \\
\hline Desgaste de reinas & 434 & 64 & 384 & 373 & 628 & 261 & 741 & 78 \\
\hline TOTAL COST. FIJO & 3,557 & 2,331 & 3,218 & 3,824 & 4,449 & 3,705 & 3,723 & 2,294 \\
\hline COSTO TOT DE PROD & 10,826 & 5,651 & 7,507 & 9,489 & 14,690 & 7,395 & 11,459 & 7,130 \\
\hline INGRESO TOTAL & 14,886 & 9,277 & 8,948 & 14,020 & 18,908 & 12,136 & 15,883 & 10,844 \\
\hline RENTABILIDAD & 4,060 & 3,626 & 1,440 & 4,530 & 4,218 & 4,741 & 4,424 & 3,714 \\
\hline
\end{tabular}

Fuente: Encuesta a productores

Por su parte, la magnitud del costo fijo de producir miel con respecto al del total de la producción representa aproximadamente el 32.9\%. Dentro de las tres principales erogaciones del citado costo, la más importante fue el valor de la depreciación en infraestructura que representó el 23.3\%; le sigue en importancia la depreciación de 
equipos de protección y trabajo que constituyó el 6.5\%; en tercer lugar se ubica el valor del desgaste de las reinas con el $4.0 \%$; por último, el valor de la depreciación de los vehículos de transporte propiedad del apicultor.

Es importante mencionar que el nivel de participación del costo fijo en el total de producción representa la principal característica que ha permitido al apicultor reducir la utilización de la capacidad instalada de la explotación ante eventos que afectan negativamente su ingreso neto, con lo cual puede continuar por un tiempo más en la actividad. Esto siempre y cuando dicha reducción no repercuta en el reembolso del capital de trabajo invertido porque de lo contrario se tendrían mayores pérdidas.

De acuerdo con lo expuesto, se puede decir que la composición porcentual del costo tiene relevancia en la administración de la producción o de las empresas apícolas del país, ya que variaciones en los precios hacia la baja afectan en forma más sensible a los apicultores cuyo costo fijo es menor. Asimismo, cuando el valor del costo fijo no es considerado (contabilizado) en el total de la producción, el efecto de su omisión se presenta cuando se requiera la reposición de los activos fijos. Este problema podría agravar aún más la falta de liquidez de los apicultores.

\section{Ingreso por venta de productos de la colmena}

Un aspecto importante del análisis del ingreso es el destino de la producción obtenida y las diferentes alternativas de venta que tiene el productor, bien sea ésta en su localidad, región, estado u otro destino. Para el presente caso, se observa del cuadro 4 que casi la totalidad de la miel cosechada en México se destina a la venta, pues una parte muy pequeña de ésta se destina al autoconsumo, la cual no rebasa en promedio el $0.7 \%$. En estados como Yucatán y Quintana Roo donde el consumo es de $1.5 \%$ se explica, generalmente, por la práctica que tienen los productores de ofrecer miel extractada a las colonias en las épocas críticas de floración; esta práctica se realiza también en todos los estados productores del país y una parte de esta miel se destina al consumo familiar. 


\section{Cuadro 4 \\ Principales destinos de la miel}

\begin{tabular}{|c|c|c|c|c|c|}
\hline \multirow[b]{2}{*}{ REFERENCIA } & \multirow{2}{*}{$\begin{array}{c}\text { AUTOCON- } \\
\text { SUMO }\end{array}$} & \multicolumn{4}{|c|}{ VENTA Y MERCADO DE DESTINO } \\
\hline & & $\mathrm{J} \cap C \wedge \mathrm{I}$ & REGIÓN & OTRO & EXPOR \\
\hline & $(\%)$ & $(\%)$ & $(\%)$ & $(\%)$ & $(\%)$ \\
\hline PROMEDIO EST. & 0.7 & 12.2 & 59.3 & 20.4 & 7.4 \\
\hline CAMPECHE & 0.4 & 6.2 & 93.2 & 0.2 & 0.0 \\
\hline CHIAPAS & 0.2 & 57.2 & 24.5 & 17.5 & 0.5 \\
\hline GUERRERO & 0.9 & 42.1 & 52.2 & 3.1 & 1.7 \\
\hline JALISCO & 0.7 & 13.5 & 29.1 & 41.6 & 15.1 \\
\hline QUINTANA ROO & 1.5 & 10.2 & 64.7 & 23.5 & 0.0 \\
\hline VERACRUZ & 0.2 & 7.9 & 88.9 & 0.3 & 2.7 \\
\hline YUCATÁN & 1.5 & 6.5 & 91.0 & 1.0 & 0.0 \\
\hline
\end{tabular}

Fuente: Encuesta a productores

De la miel que se destina a la venta en México, la mayor parte se canaliza hacia los mercados de la región, representados principalmente por los lugares o centros de acopio de los comisionistas de mayoristas, intermediaros independientes y mayoristas que operan en el estado. En este mercado, como en el local o el de los otros estados, el precio que se le paga al apicultor se determina, por lo general, por el mayorista exportador o por el mayorista que le vende a algún comercializador internacional o broker, en esto influye tanto lo que ocurre con la demanda en el mercado internacional (importación), como con el margen de ganancia que busca dicho agente comercial; es decir, el apicultor no tiene poder de negociación en la mayoría de los mercados.

Por otra parte, el valor que obtiene el apicultor nacional por la venta de la miel representa aproximadamente el $97.3 \%$ del ingreso total por la venta de los productos de la colmena que son enviados al mercado. Esta tendencia se presenta en todos los estados de interés; por ejemplo, en Chiapas, Campeche y Quintana Roo esta proporción supera el 99\%, mientras que en Jalisco, entidad con la menor proporción, ésta es de $95.4 \%$. Estas cifras revelan que la producción y venta de otros productos obtenidos de la colmena es escaso, lo cual coincide con la vocación primaria de esta actividad, en la que la diversificación productiva se limita a miel y cera; este último producto generalmente lo destina el apicultor a la sustitución de los panales, cuya venta en greña es escasa. 


\section{Cuadro 5 \\ Ingreso obtenido por la venta de miel por apiario}

\begin{tabular}{ccccc}
\hline \multirow{2}{*}{ REFERENCIA } & \multicolumn{4}{c}{ VALOR Y LUGAR DE VENTA DE LA MIEL } \\
\cline { 2 - 5 } & $\begin{array}{c}\text { LOCAL } \\
(\$)\end{array}$ & $\begin{array}{c}\text { REGIÓN } \\
(\$)\end{array}$ & $\begin{array}{c}\text { OTRO EDO. } \\
(\$)\end{array}$ & $\begin{array}{c}\text { EXPORTACIÓN } \\
(\$)\end{array}$ \\
\hline PROMEDIO EST. & 2,640 & 7,910 & 2,961 & 968 \\
CAMPECHE & 542 & 8,626 & 29 & 0 \\
CHIAPAS & 4,309 & 2,852 & 1,698 & 63 \\
GUERRERO & 7,598 & 5,668 & 271 & 215 \\
JALISCO & 3,956 & 4,967 & 6,864 & 2,260 \\
QUINTANA ROO & 1,486 & 6,705 & 3,825 & 0 \\
VERACRUZ & 2,198 & 13,135 & 42 & 411 \\
YUCATÁN & 459 & 10,150 & 78 & 0 \\
\hline
\end{tabular}

Fuente: Encuesta a productores

De acuerdo con la estructura de valor de la venta de miel por destino que se presenta en el cuadro 5, y como es de esperarse, éste es mayor cuando se vende en la región, pero no por ello se obtiene el mayor precio, pues al considerar como referencia el precio promedio obtenido en la venta (cuadro 6), se constata que el mercado de la región es donde se paga el menor precio. Lo anterior se explica por la presencia de los principales agentes comerciales que operan en este mercado (comisionistas de mayoristas e intermediarios independientes) que simplemente toman como precio base de su transacción el precio que determina el mayorista al que le van a entregar o vender la miel.

\section{Cuadro 6}

\section{Precio promedio pagado al apicultor en la venta de miel}

\begin{tabular}{ccccc}
\hline \multirow{2}{*}{ REFERENCIA } & \multicolumn{4}{c}{ PRECIO PAGADO SEGUN MERCADO DE DESTINO } \\
\cline { 2 - 5 } & $\begin{array}{c}\text { LOCAL } \\
(\$ / \mathrm{kg})\end{array}$ & $\begin{array}{c}\text { REGIÓN } \\
(\$ / \mathrm{kg})\end{array}$ & $\begin{array}{c}\text { OTRO EDO. } \\
(\$ / \mathrm{kg})\end{array}$ & $\begin{array}{c}\text { EXPORTACIÓN } \\
(\$ / \mathrm{kg})\end{array}$ \\
\hline PROMEDIO EST. & 30.8 & 19.9 & 20.4 & 23.5 \\
CAMPECHE & 20.5 & 18.4 & 19.0 & \\
CHIAPAS & 19.3 & 22.7 & 22.2 & 30.0 \\
GUERRERO & 29.0 & 19.4 & 16.0 & 26.0 \\
JALISCO & 34.1 & 21.8 & 20.1 & 22.3 \\
QUINTANA ROO & 23.2 & 18.5 & 19.9 & \\
VERACRUZ & 35.1 & 20.6 & 18.0 & 21.7 \\
YUCATÁN & 18.1 & 19.5 & 18.9 & \\
\hline
\end{tabular}

Nota: Precio promedio ponderado de 2007 y 2008

Fuente: Encuesta a productores 
De la información presentada en el cuadro anterior, se observa que el mercado local, el cual incluye la venta directa a las familias o al consumidor final, representa la mejor opción por precio para las transacciones comerciales que realizan los apicultores del país, pero este mercado no tiene la capacidad de compra que tiene el regional, o el catalogado como de otro estado, ambos manejados principalmente por comisionistas de mayoristas de las mismas empresas comerciales que operan en el ámbito nacional, cuyos precios son muy similares y, no por coincidencia, los más bajos; aproximadamente el $80 \%$ de la miel se canaliza a dichos destinos comerciales.

Con base en lo ya expuesto, se puede afirmar que la causa principal que puede provocar la variación en el nivel de la rentabilidad de las explotaciones apícolas en México lo constituye la caída del ingreso por venta, el cual se asocia al precio que se le paga al productor y, como se observa, éste depende del mercado donde se realice la transacción.

\section{Rentabilidad de la actividad apícola}

Como ya se ha mencionado, una medida de la competitividad del sistema o proceso de producción empresarial es su nivel de rentabilidad que, dado el nivel de precios de los insumos y productos y los efectos de la política macroeconómica y sectorial, permite su presencia o continuidad en el mercado. De esta manera, la magnitud de la rentabilidad indica el grado de competitividad que alcanza una empresa bajo las condiciones prevalecientes de la economía. Por lo tanto, un sistema que no genera un nivel aceptable de ganancia es un sistema que desaparecerá del mercado y, por el contrario, cuando el sistema es capaz de generar un coeficiente de ganancia superior al de referencia en la economía (costo de oportunidad del capital) éste continuará en la actividad con opción de incrementar su tamaño, mejorar su nivel tecnológico y de integrarse en forma eficiente a las diversas redes de valor, necesidad en una economía en desarrollo.

La rentabilidad de la producción apícola se presentó en el cuadro 3 y en él se observa que el valor promedio de ésta es positivo en todos los estados de interés, pero cuando se segmenta o estratifica a los apicultores nacionales por su tenencia de colmenas (cuadro 7), se observa que la rentabilidad se incrementa a medida que aumenta el número de colmenas en posesión, pero en los niveles más altos de tenencia la rentabilidad inicia un descenso. Este fenómeno que se observa en la actividad apícola nacional es propio de las economías de escala o por tamaño, en 
la cual al crecer en la empresa empiezan a surgir diversas ineficiencias operativas o administrativas que impactan tanto en los costos como en los ingresos, lo que explica este comportamiento.

\section{Cuadro 7}

Costo de producción y rentabilidad de la actividad apícola por apiario según tenencia de colmenas

\begin{tabular}{|c|c|c|c|c|c|c|}
\hline \multirow{2}{*}{ CONCEPTO } & \multicolumn{6}{|c|}{ SEGMENTO DE PRODUCTOR POR NÚMERO DE COLMENAS } \\
\hline & $1-20$ & $21-50$ & $51-100$ & $101-250$ & $251-500$ & $\begin{array}{l}\text { MÁS DE } \\
500\end{array}$ \\
\hline \multicolumn{7}{|l|}{ COSTO VARIABLE (\$) } \\
\hline Azúcar & 130.72 & 132.97 & 142.15 & 136.92 & 161.75 & 210.98 \\
\hline Otros insumos alim. & 9.17 & 11.32 & 7.21 & 5.74 & 10.64 & 24.41 \\
\hline Medicamentos & 54.71 & 43.10 & 27.07 & 22.61 & 33.62 & 20.98 \\
\hline Cera & 77.62 & 64.40 & 56.26 & 40.83 & 34.97 & 29.31 \\
\hline Mano de obra & 111.78 & 174.36 & 321.61 & 522.59 & 466.60 & 675.50 \\
\hline Flete & 67.82 & 57.39 & 21.15 & 8.12 & 2.24 & 0.00 \\
\hline Combustible & 67.69 & 84.23 & 154.79 & 185.79 & 245.99 & 191.21 \\
\hline Refacciones y mant. & 36.45 & 40.71 & 80.73 & 97.98 & 88.88 & 81.08 \\
\hline Otros & 2.14 & 3.27 & 2.86 & 6.48 & 19.50 & 13.25 \\
\hline TOTAL COST. VAR. & 558.10 & 611.74 & 813.83 & $1,027.05$ & $1,064.18$ & $1,246.73$ \\
\hline \multicolumn{7}{|l|}{ COSTO FIJO (\$) } \\
\hline Deprecia. equipo & 201.05 & 105.59 & 80.86 & 87.08 & 89.53 & 81.37 \\
\hline Deprecia. infraest & 339.08 & 311.03 & 285.51 & 292.31 & 244.43 & 306.19 \\
\hline Deprecia. vehículo & 34.15 & 30.34 & 33.56 & 37.23 & 23.80 & 21.49 \\
\hline Desgaste de reinas & 19.75 & 27.32 & 55.60 & 69.66 & 75.90 & 67.42 \\
\hline TOTAL COST. FIJO & 594.03 & 474.28 & 455.54 & 486.28 & 433.65 & 476.47 \\
\hline COSTO TOT DE PROD & $1,152.13$ & $1,086.02$ & $1,269.37$ & $1,513.33$ & $1,497.83$ & $1,723.21$ \\
\hline TOTAL DE INGRESOS & $1,700.13$ & $1,828.67$ & $2,003.93$ & $2,003.96$ & $2,080.14$ & $2,066.91$ \\
\hline RENTABILIDAD & 548.00 & 742.65 & 734.56 & 520.68 & 582.32 & 343.71 \\
\hline
\end{tabular}

Fuente: Encuesta a productores

Por otra parte, un hecho relevante que se observa en las pequeñas explotaciones apícolas o en las empresas del sector agropecuario autoadministradas es que en la práctica el apicultor nacional, cuando estima su rentabilidad, por lo general, no considera el costo fijo y, como solamente contabiliza los costos variables, cuando 
llega el momento de la reposición de los equipos e infraestructura no cuenta con el dinero o fondo suficiente para ello debido a que no existió el ahorro correspondiente (depreciación). Por lo tanto, esta situación provoca serios problemas de liquidez al productor en muy corto plazo, que por lo general tiene repercusiones en las innovaciones, o cambio tecnológico, que exige el desarrollo de la actividad tanto para adaptarse a los diversos cambios, como para incrementar la productividad.

Un aspecto interesante del análisis de la rentabilidad de los sistemas o procesos de producción es la magnitud de los indicadores y coeficientes derivados de sus principales componentes, los cuales permiten observar tanto la recuperación esperada de la inversión como el tipo de estrategia administrativa que siguen los productores ante eventos que, como ya fue mencionado, afectan en diferente magnitud la ganancia o rentabilidad.

\section{Cuadro 8}

\section{Indicadores de rentabilidad en la apicultura}

\begin{tabular}{lccc}
\hline REFERENCIA & $\begin{array}{c}\text { POR COL } \\
\text { MENA } \\
(\$)\end{array}$ & $\begin{array}{c}\text { POR MIL PE- } \\
\text { SOS DE INV } \\
(\$)\end{array}$ & $\begin{array}{c}\text { RELACIÓN } \\
\text { BENE/COST }\end{array}$ \\
\hline PROMEDIO ESTATAL & 158.0 & 305.3 & 0.375 \\
CAMPECHE & 171.1 & 383.5 & 0.642 \\
CHIAPAS & 83.7 & 164.8 & 0.192 \\
GUERRERO & 180.5 & 381.9 & 0.477 \\
JALISCO & 123.7 & 215.9 & 0.287 \\
QUINTANA ROO & 230.1 & 409.9 & 0.641 \\
VERACRUZ & 205.8 & 477.4 & 0.386 \\
YUCATÁN & 207.5 & 451.7 & 0.521 \\
\hline
\end{tabular}

Nota: La inversión no considera el valor de vehículos

Fuente: Encuesta a productores

En el cuadro 8 se presenta información relativa a los principales indicadores y coeficientes de rentabilidad; de él se observa que la rentabilidad por colmena en el país asciende a 158 pesos, a 305.3 pesos por cada mil pesos invertidos en activos fijos y la relación beneficio-costo indica que en la apicultura por cada peso invertido (costo variable y fijo) se obtiene una rentabilidad de 38 centavos. Este último indicador evidencia que la apicultura en México sí es rentable, pues por cada peso invertido en la actividad se supera con creces el rédito de una inversión equivalente en pagarés de renta fija. 
Asimismo, con base en la información del cuadro 8, se corrobora lo comentado con respecto a la apicultura del estado de Jalisco, donde el monto de las inversiones son elevadas y, al obtener una rentabilidad parecida al de los demás estados, su indicador de rentabilidad es menor. Pero ya en valores totales la ganancia total es alta, como lo es la inversión, aunque en términos relativos éste parezca menor al de otros estados.

Con relación a la influencia que ejercen las principales variables de los dos componentes de la rentabilidad sobre ésta, permite afirmar que, con base en los parámetros estimados de la regresión, el precio de venta de la miel resulta el principal determinante en esta relación, pues la variación de un peso en este precio determina un cambio promedio de $\$ 22.5$ en la rentabilidad obtenida por colmena en producción, mientras que la segunda variable de importancia lo constituye la productividad, la cual al variar en un kilogramo por colmena la rentabilidad lo hace en $\$ 15$. Con relación a la influencia que ejercen las variables del costo de producción, se observó que del costo variable, el precio del azúcar, principal insumo empleado en la alimentación de las colonias de abejas, es el que determina la mayor variación (en sentido inverso) sobre la rentabilidad, un incremento de un peso en dicha variable provoca una reducción de \$12.8 sobre esta última; le sigue en importancia la influencia que ejercen las variaciones en el salario (\$-0.15) y en el valor del transporte (\$- 0.14). Por su parte, de los costos fijos, el valor promedio de la depreciación de la infraestructura productiva y comercial por colmena es la que ejerce una limitada influencia sobre la rentabilidad obtenida, ya que al incrementarse este valor en un peso la rentabilidad sólo se reduce aproximadamente en 1.5 pesos.

Por último, es importante mencionar que la evidencia proporcionada por el modelo de regresión indica al precio de venta, a la productividad y al precio del azúcar como las principales variables explicativas de la rentabilidad, por lo que toda acción encaminada a mejorar la situación económica debe considerar una mejor integración a los canales de comercialización, adopción de mejores prácticas y técnicas de producción, así como impulsar estructuras de organización para la adquisición de insumos a menores precios. Lo anterior confirma que para obtener una mayor rentabilidad principalmente se tendrá que incrementar los ingresos de venta de la miel. 


\section{Conclusiones}

La estructura porcentual de los costos de producción de la miel en México se compone mayoritariamente por el costo variable, con una participación relativa del $67.1 \%$, cuyos principales rubros de la estructura de estos costos son el pago de mano de obra y la compra de los insumos alimenticios. Mientras que en el caso de los costos fijos, su magnitud con respecto al del total de la producción representa el 32.9\%; su principal erogación es el valor de la depreciación en infraestructura que representó el $23.3 \%$.

La composición porcentual del costo tiene relevancia en la administración de la producción apícola del país, ya que variaciones en los precios hacia la baja afectan en forma más sensible a los apicultores cuyo costo fijo medio es menor. Asimismo, cuando el valor del costo fijo no es contabilizado en el total de la producción, el efecto de su omisión se presenta cuando se requiere la reposición de los activos fijos; práctica común en casi la totalidad de los apicultores de México.

La causa principal que puede provocar la variación en el nivel de la rentabilidad de las explotaciones apícolas en el territorio nacional lo constituye la caída del ingreso por la venta de miel, el cual determina el precio que se le paga al productor mexicano y tal precio depende del mercado donde se realice la transacción. En tanto que la rentabilidad por colmena en México asciende a 158 pesos y a 192.2 pesos por cada mil pesos invertidos en activos fijos, en tanto que la relación beneficiocosto indica una rentabilidad de 38 centavos por cada peso invertido. Esto último evidencia que la apicultura es rentable, pues lo invertido en la actividad supera la recuperación de una inversión equivalente en pagarés de renta fija.

Con base en los resultados obtenidos y por la experiencia obtenida en la investigación de campo, se puede afirmar que la actividad apícola presenta tres principales oportunidades de desarrollo para mejorar su rentabilidad, a saber:

1. Producción de miel orgánica. Con ésta se incrementa la calidad y aceptación de los productos apícolas y, en consecuencia, sus respectivos precios en el mercado; estos productos son los más cotizados en los países europeos, como Alemania. Esta oportunidad permite incrementar la rentabilidad y alcanzar una mayor competitividad en los mercados. 
2. Agregar valor a los productos de la colmena. Esta proporciona una alternativa más para la comercialización, pues por medio de la industrialización de los productos primarios se obtienen diversos productos finales, entre los que están los coadyuvantes en el tratamiento de algunos padecimientos respiratorios, suplementos alimenticios, golosinas, cosméticos, entre otros, que se orientan a satisfacer la demanda de los consumidores internos y, con los canales adecuados, la de los externos. El valor agregado incrementaría las utilidades y, en consecuencia, se dinamizaría toda la una cadena productiva y comercial.

3. Integración adecuada a los canales de comercialización. Con ésta se favorecería la relación directa con los principales compradores de otros eslabones de la cadena productiva o comercial (agroindustriales, comercializadores y distribuidores) y, como consecuencia, permitiría conseguir mejores precios e ingresos al eliminar la participación de los agentes comerciales innecesarios.

4. La apicultura puede considerarse como una oportunidad de negocios siempre y cuando se garantice la rentabilidad buscando alternativas que permitan incrementar los ingresos por medio del incremento de la productividad actual y definiendo mejores estrategias de comercialización.

Finalmente, a pesar de todas las vicisitudes que se presentan en la actividad apícola, ésta es aún rentable, ha mantenido su importancia social y económica y, con relación al exterior, es aún competitiva.

\section{Referencias}

Alonso, S. R. y B. A. Serrano (1991). Los costes en los procesos de producción agraria. Metodología y aplicaciones, Madrid: Ediciones Mundi-Prensa.

Centro Regional de Estudios Económicos de Bahía Blanca (CREebBa) (2005). La rentabilidad de la apicultura. Estudios especiales, indicadores de actividad económica, pp.12-18. dsiponible en: www.creebba.org.ar.

FAOSTAT 2009. Base de información estadística en línea de la FAO con relación a la alimentación y la agricultura. Consulta 2009 
Feitosa F. Débora, K. Silva Saeed y Lucía Ramos (2004). Nivel y producción tecnológicos de producción de la miel de la abeja (Apis mellifera) en el Ceará. RER, Río de Janeiro, Vol. 42, nº 01, enero/marzo, pp. 171-188.

Güemes, R. F., G. C. Echazarreta, G. R. Villanueva, F. J. Pat y A. R. Gómez (2003). La apicultura en la península de Yucatán. Actividad de subsistencia en un entorno globalizado, RMC, 16, pp.117-132.

Kay, R. D. (1990). Administración agrícola y ganadera. Planeación, control e implementación. A. G. Mendoza, México: Compañía Editorial Continental.

Red Nacional de Centros de Gestión y el Instituto de Desarrollo Agropecuario de Chile (2008). Estudio económico del rubro miel a partir de información registrada por Centros de Gestión, Chile.

Scheafer, R. L., W. Mendenhall y L. Ott. (1987), Elementos de muestreo, México: Iberoamérica.

SIACON, Sistema de Información Agroalimentaria de Consulta. Información estadística en línea de la SAGARPA, México. Consulta 2009.

Stevenson, W. (1981). Estadística para administración y economía. México: HARLA.

Tiatrini. 2002. Historia de la apicultura en México. Disponible en: http://www. tiatrini.com.mx/apimex.htm, 21/10/04.

Villanueva R. y W. Collí. (1998). La apicultura en la península de Yucatán, México y sus perspectivas, México: Apitec 11.

Páginas de internet consultadas:

www.agronet.com.mx

www.InfoAgro.com, 2005 
